Contaduría y Administración 64 (1) Especial Innovación, 2019, 1-21

\title{
¿Cómo se gestiona la tecnología en las pymes? Diferencias y similitudes entre micro, pequeñas y medianas empresas
}

\author{
How is technology managed in SMEs? Differences and similarities between \\ micro, small and medium enterprises
}

\author{
Salvador Estrada ${ }^{1 *}$, Karen Cano ${ }^{1}$, Joao Aguirre $^{2}$ \\ ${ }^{1}$ Universidad de Guanajuato, México \\ ${ }^{2}$ Strategic Intelligence, Australia
}

Recibido el 26 de noviembre de 2017; aceptado el 17 de noviembre de 2018

Disponible en Internet el: 27 de noviembre de 2018

\section{Resumen}

En el ámbito de la innovación y el desarrollo tecnológico, las pequeñas y medianas empresas (Pymes) muchas veces no cuentan con los recursos necesarios para iniciar proyectos. El comportamiento innovador y tecnológico está condicionado por una serie de elementos, tales como la estructura, los recursos financieros, el entorno y el sector. Cada uno presenta condiciones de competencia que predeterminan sus posibles estrategias y conducta. Para una eficiente gestión de la tecnología es menester considerar las oportunidades y amenazas de su posición tecnológica, la capacidad de adquirir y desarrollar recursos, la habilidad para asimilar las tecnologías y el aprender de esta experiencia. Las Pymes establecen en la práctica diversos modelos. El presente trabajo pretende caracterizar las diferentes funciones llevadas a la práctica a partir de ciertos modelos establecidos en la literatura. A partir de una muestra representativa de 81 empresas y mediante un análisis de conglomerados, se lograron identificar 6 diferentes modelos empíricos definidos por diferencias y similitudes en el análisis del entorno, investigación de mercados y

\footnotetext{
*Autor para correspondencia.

Correo electrónico: salvador.estrada@gmail.com (S. Estrada)

La revisión por pares es responsabilidad de la Universidad Nacional Autónoma de México.
} 
evaluación de tecnología, adquisición y adaptación de la tecnología y asimilación e implementación de tecnología, los cuales están mediados por el tamaño, el sector y la localización.

Códigos JEL: O3, D41

Palabras clave: Pequeñas empresas, conducta tecnológica, innovación.

\begin{abstract}
In the field of innovation and technological development, regularly small and medium enterprises (SMEs) do not have the required resources to start projects. The innovative and technological behavior is conditioned by several elements, such as structure, financial resources, the environment and the sector. Each one presents competitive conditions that predetermine their possible strategies and behavior. For an efficient management of technology, it is necessary to consider the opportunities and threats of its technological position, the capability to acquire and develop resources, the capability to assimilate technologies and learn from this experience. In practice, the SMEs establish different models. The present work aims to characterize the different functions carried out from certain models established in the literature. From a representative sample of 81 companies and through a cluster analysis, we were able to identify 6 different empirical models defined by differences and similarities in the analysis of the environment, market research and technology assessment, acquisition and adaptation of technology, assimilation and technology implementation, which are mediated by size, sector and location.
\end{abstract}

JEL Codes: M10, M 20, O30

Keywords: Learning orientation; Innovation; Firm performance.

\title{
Introducción
}

Para México las Pymes representan un sector en crecimiento debido a que contribuyen con la producción de alrededor del 32\% del Producto Interno Bruto (PIB). La importancia económica es obvia, sin embargo al ser un sector de la economía muy disperso y diversificado, aún existe mucha volatilidad en la creación y crecimiento de estas empresas. Según datos de Adecco (2016), las probabilidades de éxito en el mercado local están en rango promedio de entre $25 \%$ y $30 \%$ debajo de la media mundial que es del $40 \%$.

Bajo una perspectiva estratégica, los recursos y capacidades desarrollados por la PYMES sufren por la escasez de habilidades, pobres prácticas gerenciales y una limitada formación y entrenamiento de su mano de obra. Tampoco están muy expuestas a las mejores prácticas globales de calidad, diseño o producción (Farid, 2017). Esta situación se traduce en un desempeño variable en su rentabilidad, supervivencia y crecimiento.

En los ámbitos de Innovación e Investigación y Desarrollo Tecnológico, las Pymes se ven afectadas debido a que muchas veces no cuentan con los recursos necesarios para iniciar proyectos de I\&D. Según un reporte reciente del BID (2010): "Un número sustancial de empresas en la región se definen como innovadoras y practican la innovación de una u otra manera". Según diversas encuestas realizadas en Ibero-América es considerable la tasa de empresas innovadoras en procesos (aproximadamente en el rango 15-40\%) (Peirano, 2007; Anlló y Suárez, 2008, Marins, Anlló y Schaaper, 2012). Sin embargo, sigue existiendo una amplia brecha tecnológica con los países líderes y muchas veces estos esfuerzos innovadores representan una respuesta al creciente desafío competitivo derivado de la globalización. 
En una mayoría de casos, las Pymes en América Latina han realizado una gestión tecnológica para atender los requerimientos de los recursos tecnológicos básicos o genéricos que permitan ingresar y mantenerse en el mercado por lo cual no existe ni una estructura funcional encargada, ni se desarrollan planes o agendas tecnológicas además de carecer de presupuestos específicos para estas actividades (Malaver y Vargas, 2004).

La literatura reciente sobre gestión tecnológica en Pymes destaca el esfuerzo por generar marcos de evaluación sobre el tipo de estrategia implementada (Bolukbas y Guneri, 2017), así como los impactos de esta actividad sobre el desempeño empresarial en términos de reducción de costos (Burggräf, Dannapfel, Bertling y Xu, 2018; , diferenciación (Loon y Chik, 2018), respuesta a clientes (Zhu, Zou y Zhang, 2018; Hills y Atkinson, 2016), eficiencia (Lohmüller y Petrikhin, 2018), desarrollo de talento y competencias (Bolukbas y Guneri, 2016; Van Der Veen, Perez y Sabbatinelli, 2016; Nordin y Adegoke, 2015), integración y cooperación (Noh y Lee, 2015), pero en particular para el desarrollo de capacidades de absorción (Garengo, 2018), de adopción exitosa de tecnologías emergentes (Burggräf, Dannapfel, Bertling y Xu, 2018; Deranek, Chopra y Mosher, 2017; Niaki y Nonino, 2017), además de competencias para anticipar aplicaciones promisorias y desarrollos futuros (Bullinger, Bauer, Ardilio y Seidenstricker, 2015).

Ante un entorno crecientemente turbulento y altamente competitivo se plantea si la intención informal de la estrategia puede avanzar hacia un manejo más formal de la tecnología, desarrollo de capacidades funcionales y de prácticas constitutivas del patrimonio tecnológico, eslabonamientos y salvaguardas del conocimiento. Por lo tanto, el objetivo de este trabajo de investigación es realizar un estudio de la gestión tecnológica en las Pymes de México y en específico del estado de Guanajuato con el fin de conocer su situación actual. Para llevarlo a cabo, el presente documento se organiza de la siguiente manera: en la presente sección se hace una introducción al tema, en la segunda sección se elabora el marco teórico, a continuación, se desarrolla la metodología para dar paso, en la cuarta sección, a los resultados y, en la última parte, se presentan las discusiones y conclusiones. A partir de cuatro funciones básicas de la gestión tecnológica en pymes, se encuentran seis patrones que expresan diferencias en tamaño y localización y revelan diversos grados de sofisticación, con lo que se puede adelantar una proposición sobre un patrón dinámico de crecimiento con base en las prácticas de gestión tecnológica.

\section{Marco teórico}

La gestión tecnológica surgió como respuesta a la necesidad de manejar el factor tecnológico con un sentido estratégico. Se define como el proceso de manejar todas aquellas actividades que habiliten a la empresa para hacer el uso más eficiente de la tecnología generada internamente y de la adquirida a terceros, así como de incorporarla a los nuevos productos (innovación de producto) y a las formas en que los producen y se entregan al mercado (innovación de proceso). Se encarga de los problemas de decisión a todos los niveles relacionados con la creación y utilización de activos y capacidades tecnológicas; sus impactos sobre los individuos, organizaciones, sociedades y naturaleza; y la conciliación de las consecuencias económicas, sociales y ambientales de las innovaciones tecnológicas (Bayraktar, 1990).

La gestión tecnológica busca mejorar las capacidades de absorción y de internalización del conocimiento para lo cual puede auxiliarse de metodologías que apoyen estas actividades 
(Medellín, 2010). Por tanto, los modelos de gestión constituyen herramientas para la toma de decisión sobre estrategias innovadoras (Mendoza y Valenzuela, 2014). A continuación, se comentan seis modelos que buscan guiar una correcta práctica, los cuales pueden ser llevados a cabo tanto por Pymes como por grandes empresas.

(01) El modelo del PNT (Premio Nacional de Tecnología e Innovación, 2012) tiene como objetivo principal impulsar el desarrollo de las organizaciones de cualquier giro o tamaño, para proyectarlas de manera ordenada a niveles competitivos de clase mundial mediante la gestión tecnológica explícita sostenida y sistemática. Se compone de una serie de procesos y funciones.

(02) El modelo COMECYT (Cuevas, León y Ramírez, s.f.) establece como solución permanente para la elaboración de productos y servicios basarse en la capacidad de organización y habilidades para crear, almacenar, transferir, aplicar y difundir el conocimiento a través de redes formales. El modelo se representa con una espiral cuya parte central es la innovación, brindando esencial importancia a la creatividad, calidad, valor agregado, y actitud emprendedora. Este modelo debe ser una herramienta de coordinación para el capital humano, además de ser un medio para fijar y alcanzar las metas en el desarrollo de los productos.

(03) El modelo Sumanth y Sumanth (1996) propone un enfoque sistémico de la gestión tecnológica, mediante un proceso continuo que puede ser aplicado al producto, servicio, centro de trabajo y corporación nacional o internacional.

(04) En el modelo de Hidalgo, León y Pavón (2002) se definen un conjunto de procesos de gestión específicos, adaptados a la tecnología, para identificar, evaluar, seleccionar, adquirir, asimilar y utilizar eficientemente la tecnología. Cada sector empresarial deberá ajustar estos procedimientos a sus necesidades.

(05) El modelo COTEC (1998) (Fundación para la Innovación Tecnológica Española, la empresa SOCINTEC, CENTRIN (Universidad de Brighton), IRIM (Universidad de Kiel) y la Unidad de I+D de la Manchester Business School ) está basado en una estructura poco compleja que abarca cinco elementos o funciones. Cada elemento puede auxiliarse de diversas herramientas para su realización como puede ser la investigación de mercado, el análisis FODA, la prospectiva y el pronóstico tecnológico, la inteligencia competitiva, la cadena de valor, la evaluación y gestión proyectos, la gestión de cartera, diagrama de causa/efecto, gestión de la propiedad intelectual, análisis de valor, gestión del cambio o la gestión del talento y del conocimiento, entre otras.

(06) Antonio Hidalgo (1999) expone en su modelo a la gestión tecnológica como un factor estratégico de la competitividad industrial. Acentúa cuáles son las funciones, así como también las herramientas esenciales del proceso de la gestión tecnológica. El modelo clasifica las funciones para desarrollar la gestión tecnológica eficientemente en activas y de apoyo.

En resumen, de los 6 modelos investigados encontramos etapas y /o actividades en común (entre paréntesis el modelo al cual se hace referencia) como son:

- Análisis del entorno: (Anticipar la eficiencia, la optimización y la simplificación)

(01) (03) (05) Vigilancia del entorno mediante un proceso organizado, formal, selectivo y sistemático a fin de tomar decisiones con menor riesgo y anticipar cambios.

(04) (06) Identificar el potencial tecnológico propio en términos de necesidades del mercado y competidores principales.

(02) Discusión de la idea con los socios tecnológicos, de capital y operativos.

- Investigación de mercado y evaluación de la tecnología (desarrollar criterios para mejorar la competitividad) 
(01) (02) (03) (04) (05) Seleccionar las tecnologías más adecuadas, contemplando factores como la disponibilidad, el costo, la relación con otras tecnologías etc.

(01) (05) (06) Focalizar recursos identificando tecnologías críticas, seleccionar líneas de acción y elaborar plan tecnológico.

- Adquisición y adaptación de la tecnología (despliegue de herramientas técnicas y disposición de los recursos tecnológicos)

(01) (02) Obtención, dentro y fuera de la organización, de tecnologías y recursos necesarios

(04) Identificar y evaluar proveedores, y suministros.

(06) Incrementar el patrimonio tecnológico (examinar las posibilidades externas antes de decidirse por realizar el desarrollo internamente).

(02) (05) Capacitarse (una vez elegida la opción tecnológica, la empresa tiene que asignar los recursos necesarios para materializar la opción elegida), incorporación de personal de investigación.

(02) Implementación de infraestructura de experimentación a nivel laboratorio y posteriormente de escalamiento a la planta industrial.

(02) Realización de los proyectos de I+D.

(02) factores exógenos como la asistencia técnica, financiamiento, la vinculación.

- Asimilación e implementación del patrimonio tecnológico (mejorar posición competitiva por acumulación e implementación de capacidades tecnológicas)

(02) (05) (06) Realización de los proyectos de innovación hasta el lanzamiento final como nuevo producto o servicio.

(02) Indicadores para medir el desempeño de la innovación, así como los impactos tecnológicos, organizacionales, financieros y sociales.

(01) (02) Salvaguarda y cuidado del patrimonio tecnológico mediante la protección de la marca y patentes.

(02) factores exógenos como la divulgación (promoción de la innovación para generar una imagen de calidad de ésta).

(03) (04) Mejora de las tecnologías adquiridas adaptándolas a sus necesidades particulares, lo que implica la formación de personal y el establecimiento de procedimientos internos.

(03) Decisiones importantes con respecto a la obsolescencia de una tecnología dada.

(05) Aprender (catalogar la experiencia de éxito o de fracaso).

De acuerdo con Faloh Bejarano (2006), un buen sistema de gestión tecnológica debe reflejarse en el modelo utilizado que, se supone, simplifica o se aproxima al sistema real. Las restricciones están relacionadas con las características de la empresa, sus estrategias, el tipo de productos o servicio que vende, márgenes de utilidad, la naturaleza de las tecnologías, el nivel de dominio que tiene de éstas, la atención al entorno, las inversiones en $\mathrm{I}+\mathrm{D}$, las actividades de innovación y su efecto en el funcionamiento de la empresa.

Las Pymes pueden ser incapaces de explotar nuevos productos debido a las limitadas capacidades organizativas y de comercialización (Cobbenhagen, 1999). Filson y Lewis (2000) y Freel (2000) analizan las barreras culturales a la innovación, como la resistencia al cambio, la tendencia a ignorar el procedimiento, centrarse en las necesidades a corto plazo, la falta de visión estratégica y la difusión de una cultura de la culpa. La tendencia a ignorar los procedimientos -para no asumir la responsabilidad-, la ausencia de actividades de supervisión de procesos y una gestión deficiente son problemas que impactan al proceso de innovación definido como 
una secuencia de tareas. El estudio de Scozzi, Garavelli y Crowston (2005) hace una revisión de la literatura sobre estas problemáticas y su incidencia sobre la innovación. La falta de vigilancia tecnológica, funciones de búsqueda y de memoria de la organización se mencionan como problemas significativos en los estudios que interpretan el proceso de innovación como un flujo de decisiones. Un problema importante, que también se considera, es la falta de una visión estratégica para impulsar el desarrollo de la innovación (Bullinger, Bauer, Ardilio y Seidenstricker, 2015). Los problemas asociados a la perspectiva creativa, tales como la existencia de una cultura de la culpa, dependen de las habilidades y capacidades del empresario, por lo que no son comunes a todas las empresas (Scozzi, Garavelli y Crowston, 2005).

En su estudio sobre gestión tecnológica en pymes de alta tecnología, Loon y Chik (2018) sugieren que hay un rango de empresas entre las que buscan eficiencia e innovación. Por otro lado, Lohmüller y Petrikhin (2018), establecen que esta gestión requiere de diversos roles de la gerencia por lo que su diferente implicación originaría diversos arreglos y resultados. En este mismo sentido, pero enfatizando las capacidades gerenciales e incorporando además las infraestructuras tecnológicas Bulokbas y Guneri (2018), encuentran para el caso de las pymes manufactureras turcas diversos niveles de competencias tecnológicas.

De aquí podemos desprender una proposición para el estudio que abordaremos:

P1. Las Pymes del estado de Guanajuato del sector industrial, conforman diversos patrones empíricos de la gestión de tecnología de acuerdo con su tamaño, sector y localización.

\section{Metodología}

Tomando como base la literatura de gestión de tecnología, es decir, desde la definición y los criterios esenciales para la práctica de ésta, incorporando a su vez las actividades y fases de los modelos anteriormente mencionados, se pretende conocer mediante el presente estudio si las actividades realizadas son más apegadas a alguno de los seis modelos o si las pymes realizan esta actividad de manera empírica.

Se realizó una investigación empírica con un enfoque cuantitativo de tipo explicativo y corte transversal, a través del Análisis de la Varianza (ANOVA) y el Análisis de conglomerados jerárquicos (HCA). En este estudio se analizó la gestión tecnológica en MiPymes industriales, utilizando las variables tamaño, sector y localización como control. De igual manera se aplicó al ANOVA la prueba de Tukey, con la intención de encontrar las fuentes de las diferencias estadísticamente significativas entre los grupos, tomado como referencia el factor tamaño de las empresas objeto de estudio.

En los estudios de innovación en Pymes, el análisis clúster ha sido muy útil para determinar diferencias en la percepción empresarial de barreras a la innovación (Marin, Marzucchi y Zoboli, 2015), fuentes de suministro de información y conocimiento en la innovación abierta (Brunswicker y Vanhaverbeke, 2015) el grado de apertura (Othman Idrissia, Amaraa y Landrya, 2012), el nivel de uso de comercio electrónico (Sila y Dobni, 2012), actividades de marketing y ventas en las estrategias de producto (Cant, Wiid y Kallier, 2015) así como el nivel de competencia tecnológica (Bolukbas y Guneri, 2018).

\section{Diseño de la muestra y recolección de datos}

Para el desarrollo de este trabajo de investigación se tomó de referencia la base de datos que ofrece el Sistema de Información Empresarial Mexicano (2013), en el que aparecen registradas 
en el estado de Guanajuato hasta el 25 de noviembre del 2013, un total de 15,507 empresas de los sectores agrícola, construcción y manufacturero de los municipios de León, Silao, Guanajuato, Irapuato, Salamanca y Celaya, de 1 a 250 trabajadores, universo que se reduce con el criterio de exclusión de que tengan correo electrónico a 951 empresas.

Es por ello que, la encuesta se diseñó en base a los modelos teóricos analizados, así como información general de la empresa, para que fuera contestada por los gerentes o dueños de las MiPymes del sector industrial de Guanajuato - dado el caso de que no pudieran atender la encuesta, ésta también fue tomada por personal de administración y/o gerentes de producción-, y fue aplicada de manera aleatoria, con un nivel de confianza del $95 \%$ y un margen de error del $5 \%$, por lo que se enviaron un total de 284 encuestas y fueron aplicadas en tres oleadas en el periodo diciembre del 2013 a agosto de 2014.

La herramienta utilizada para la recolección de datos fue mediante un software en línea llamado SURVEYMONKEY, donde por medio del correo electrónico se les envió a los encuestados una invitación de participación. Algunos otros fueron por medio de llamadas telefónicas y asistencias programadas por medio de citas a las empresas. Las respuestas fueron vaciadas al software de análisis estadístico SPSS V.17. Se obtuvo una tasa de respuesta del $30 \%$, contando al final con un total de 81 encuestas válidas.

En la Tabla 1 se muestran las distribuciones de la muestra por sector, estrato de tamaño según el número de empleados y localización. Como puede verse el sector mejor representado es el de pequeñas empresas con $42 \%$, mientras que sectorialmente es el de construcción que llega hasta casi al 30\% mientras que Agroindustria es de $11.1 \%$ y Manufactura del 58\%. La composición de la muestra guarda cierta consistencia con la especialización de la región.

Tabla 1.

Distribución de la muestra por tamaño, sector y localidad de actividad

\begin{tabular}{lcc}
\hline Tamaño & Número de empresas & Porcentaje \\
\hline Micro & 26 & 32.1 \\
Pequeña & 34 & 42 \\
Mediana & 21 & 25.9 \\
Total & 81 & 100 \\
\hline Sector & Número de empresas & Porcentaje \\
\hline Industria alimentos, bebidas y tabaco & 11 & 13.6 \\
Textiles y prendas de vestir & 3 & 3.7 \\
Industria de la madera y productos de madera & 2 & 2.5 \\
Productos de Papel, imprentas y editoriales & 1 & 1.2 \\
Sustancias químicas y derivados del petróleo & 2 & 2.5 \\
Industria metálica básica & 17 & 20.9 \\
Productos metálicos maquinaria y equipo & 11 & 13.6 \\
Construcción & 25 & 30.9 \\
Agroindustria & 9 & 11.1 \\
Total & 81 & $100 \%$ \\
\hline Municipio & Número de empresas & Porcentaje \\
\hline Celaya & 17 & 21 \\
Irapuato & 11 & 13.6 \\
León & 26 & 32.1 \\
Guanajuato & 9 & 11.1 \\
Salamanca & 16 & 19.8 \\
Silao & 2 & 2.5 \\
\hline Total & 81 & 100 \\
\hline Fuente: Elaboracion propia. & & \\
\hline
\end{tabular}

Fuente: Elaboración propia. 


\section{Medición de variables}

Con la revisión de la literatura efectuada se elaboró la operacionalización de variables y establecimos que los modelos teóricos se pueden sintetizar en cuatro funciones: Análisis del entorno, Investigación de mercado y evaluación de la tecnología, Adquisición y adaptación de la tecnología y Asimilación e implementación del patrimonio tecnológico.

A partir de la similitud de las fases de los modelos previamente mencionados y detallados, se preparó un cuestionario con 4 secciones. Después de la sección de introducción donde se presentan objetivos y alcances de la investigación, el empresario comienza las secciones temáticas. En la siguiente tabla se muestra la composición de la encuesta donde mencionan las variables constituyentes de cada sección, así como el tipo de variable utilizada.

Tabla 2 .

Conformación de la encuesta.

\begin{tabular}{lcc}
\hline Secciones & Variables que lo componen & Tipo de variable \\
\hline Datos generales de la empresa & $\begin{array}{c}\text { Nombre de la empresa, dirección, correo } \\
\text { electrónico, subsector, antigüedad, naturaleza }\end{array}$ & Nominal \\
\hline $\begin{array}{l}\text { Entorno y necesidades de la } \\
\text { empresa }\end{array}$ & $\begin{array}{c}\text { Elementos para análisis del entorno, fuentes de } \\
\text { información, frecuencia de análisis del entorno. }\end{array}$ & Ordinal \\
\hline $\begin{array}{l}\text { Adquisición y adaptación del } \\
\text { patrimonio tecnológico }\end{array}$ & $\begin{array}{c}\text { Tipos de tecnología, criterios para adquirir } \\
\text { tecnología, áreas de integración de I+D, recursos } \\
\text { humanos, financiamiento, infraestructura. }\end{array}$ & Ordinal \\
\hline $\begin{array}{l}\text { Asimilación e implementación } \\
\text { de la tecnología }\end{array}$ & $\begin{array}{c}\text { Fuente de los cambios, motivación de los cam- } \\
\text { bios, impacto de los cambios, ciclo de vida de }\end{array}$ & Ordinal \\
& tecnología, reputación de la empresa. & \\
\hline
\end{tabular}

Nota: Para las variables métricas e utilizó una escala perceptual de Likert para indicar frecuencia o importancia de la actividad.

\section{Análisis estadístico}

Se obtuvieron las distribuciones de frecuencias de cada variable para la muestra y submuestras por tamaño. Se agruparon de acuerdo con las fases tecnológicas. Para encontrar diferencias significativas se aplicó Análisis de la Varianza. Se aplicó la prueba de Tukey para determinar el tamaño de origen.

Para profundizar aún más y determinar con certeza cómo es el manejo de la gestión tecnológica entre las micro, pequeñas y medianas empresas (MiPymes) se aplicó un método estadístico que manifiesta el comportamiento de un grupo en base a las similitudes que presentan los casos. Mediante un análisis clúster se clasificó la muestra en un número pequeño de grupos de forma que las observaciones pertenecientes a un grupo fueron muy similares entre sí y muy disimilares del resto. Para obtener el número de grupos a analizar se utilizó el método de Ward donde se obtuvieron 6 conglomerados o clústeres.

\section{Resultados}

Se explican a continuación una serie de tablas-resumen presentadas por etapas de gestión tecnológica. Se indican los porcentajes más altos de cada variable -en términos de mayor frecuencia o importancia-, así como las variables que presentan diferencias significativas entre tamaño de empresa. 
Tabla 3.

Etapa 1. Análisis del entorno.

\begin{tabular}{llllr}
\hline Análisis interno & Elementos del análisis externo \% & Fuentes de información más importantes & $\%$ \\
\hline $90 \%$ & Proveedores & 76.6 & Noticias de empresas & 56.8 \\
& Clientes & 75.6 & *Canales de abastecimiento y proveedores & 55.5 \\
\hline & Empleados & 74.1 & Páginas web de proveedores & 54.3 \\
& *Mercado & 67.9 & Competencia (lanzamientos de nuevos productos) 53.1 \\
\hline & *Inversores y socios & 37.1 & & \\
\hline
\end{tabular}

*Variables con diferencia significativa entre tamaño de empresas.

La primera fase de gestión tecnológica que realizan las Pymes del sector industrial del estado de Guanajuato es el Análisis del Entorno. Donde el 90\% de las Pymes realizan esta actividad interna por lo menos una vez al año. En lo que se refiere a los elementos del entorno considerados para el análisis son importantes los proveedores, los clientes, los empleados. Sin embargo, los elementos para los que se encuentran diferencias significativas entre las Pymes de acuerdo a los Análisis de Varianza (no mostrados en este artículo por brevedad) son el mercado y los inversores y socios. Siguiendo los resultados de la prueba de Tukey se revela que para las empresas medianas es más importante el mercado que para las pequeñas empresas, mientras que los inversores o socios es un elemento diferenciador con respecto a las microempresas.

En lo relativo a las fuentes de información, tienen un peso específico en el análisis del entorno, pero están un tanto diversificadas, entre fuentes públicas, específicas e inter organizativas. Para la microempresa la información obtenida de los canales de abastecimiento y proveedores es un elemento diferenciador.

Tabla 4.

Etapa 2. Investigación de mercado y evaluación de la tecnología.

\begin{tabular}{|c|c|c|c|c|c|c|c|}
\hline Actividades de I+D & $\%$ & Tipo de tecnología & $\%$ & \multicolumn{2}{|c|}{$\begin{array}{l}\text { Criterios de adquisición } \\
\text { de tecnología } \%\end{array}$} & \multicolumn{2}{|c|}{ Integración de I+D } \\
\hline Últimos 5 años & 66.7 & TIC's & 46.9 & Especialización & 54.4 & Mantenimiento & 86.4 \\
\hline Últimos 3 años & 66.7 & Transformación & 46.9 & * Estudio técnico & 53.1 & *Ventas & 84.9 \\
\hline Ultimo año & 66.7 & Manufactura & 45.7 & Precio & 49.4 & Calidad & 81.4 \\
\hline & & Proceso & 45.7 & Evaluación financiera & 47 & Producción & 80.2 \\
\hline & & Transporte & 45.7 & Competencia & 45.2 & & \\
\hline & & Empaque y envasado & 43.2 & Contexto Macroecon & ómico 35.8 & & \\
\hline
\end{tabular}

* Variables con diferencia significativa entre tamaño de empresas.

La segunda fase de Investigación de Mercado y Evaluación de Tecnología (FII) revela que dos terceras partes de las Pymes estudiadas están persistentemente realizando actividades de investigación y desarrollo (el 68\% de la muestra tiene más de seis años de establecida, y por la invariante porcentaje, notamos que las que preponderantemente declaran esta actividad son las empresas más viejas). No muestran una especial dependencia en un tipo de tecnología sino que tienen su atención diversificada sin destacar alguna en nivel de importancia. También son múltiples los conceptos que consideran para la adquisición de tecnología. En cuestión de los criterios para adquirir tecnología, existe una diferencia significativa entre los tamaños de empresas resultando que para las micros es todavía más importante realizar estudios técnicos que para las medianas y pequeñas empresas. Por otra parte, la actividad de I+D no está centralizada sino es una competencia distribuida entre las actividades de mantenimiento, ventas, calidad y 
producción. Encontrando que un elemento diferenciador para la microempresa es el nivel de integración de I+D con ventas.

Tabla 5.

Etapa 3. Adquisición y adaptación de la tecnología

\begin{tabular}{|c|c|c|c|c|c|c|c|}
\hline $\begin{array}{l}\text { Contratación } \\
\text { personal } \\
\text { actividades de } \mathbf{I}+\mathbf{D}\end{array}$ & $\begin{array}{r}\text { de } \\
\text { para }\end{array}$ & $\begin{array}{l}\text { RH destinados } \\
\text { actividades de } \\
\%\end{array}$ & $\mathbf{I}+\mathbf{D}$ & $\begin{array}{l}\text { Nivel de es } \\
\%\end{array}$ & estudios & $\begin{array}{l}\text { Tipo de activida } \\
\text { desarrollar }\end{array}$ & $\begin{array}{c}\mathrm{ad} \\
\%\end{array}$ \\
\hline $44.5 \%$ & & Licenciatura & 25.9 & Licenciatura & 90.1 & Técnica & 93.8 \\
\hline Infraestructura & $\%$ & Maestría & 11.1 & Maestría & 9.9 & Investigación & 4.9 \\
\hline $\begin{array}{l}\text { Laboratorio de } \\
\text { calidad }\end{array}$ & 14.8 & Especialidad & 9.9 & Doctorado & 0 & Apoyo & 1.2 \\
\hline $\begin{array}{l}\text { Talleres de } \\
\text { mantenimiento }\end{array}$ & 14.8 & Bachillerato & 9.9 & $\begin{array}{lr}\text { Ultima } & \text { adqui } \\
\text { de } & \text { tecn } \\
\% & \end{array}$ & $\begin{array}{l}\text { uisición } \\
\text { cnología }\end{array}$ & $\begin{array}{l}\text { Condición de } \\
\text { tecnología adquirida }\end{array}$ & $\%^{\text {la }}$ \\
\hline $\begin{array}{l}\text { Laboratorio de } \\
\text { análisis físico- } \\
\text { químico }\end{array}$ & 6.2 & Carrera técnica & 7.4 & 6 meses & 40.7 & Nueva & 85.2 \\
\hline Planta piloto & 4.9 & Doctorado & 2.5 & 1 año & 45.7 & Usada & 14.8 \\
\hline $\begin{array}{l}\text { Centros de } \\
\text { información }\end{array}$ & 3.7 & $\begin{array}{l}\text { Impactos por } \\
\text { introducción } \\
\text { tecnología }\end{array}$ & $\begin{array}{l}\text { la } \\
\text { de }\end{array}$ & 2 años & 9.9 & $\begin{array}{l}\text { Uso de apoyos } \\
\text { importancia }\end{array}$ & $\begin{array}{l}\text { según } \\
\%\end{array}$ \\
\hline $\begin{array}{l}\text { Laboratorio de } \\
\text { pruebas }\end{array}$ & 3.7 & $\begin{array}{l}\text { Aumento de la } \\
\text { productividad }\end{array}$ & 45.7 & 3 años o mas & 3.7 & $\begin{array}{l}\text { *Modernización } \\
\text { tecnológica }\end{array}$ & 17.3 \\
\hline $\begin{array}{l}\text { Fuente de obtenció } \\
\text { la tecnología }\end{array}$ & ón de & $\begin{array}{l}\text { Cambio en el } \\
\text { proceso }\end{array}$ & 39.5 & $\begin{array}{l}\text { Fuente } \\
\text { financiamiento } \\
\text { la } \\
\%\end{array}$ & $\begin{array}{r}\text { de } \\
\text { para } \\
\text { I+D }\end{array}$ & $\begin{array}{l}\text { Capacitación } \\
\text { productividad }\end{array}$ & 17.3 \\
\hline Proveedores & 87.7 & $\begin{array}{l}\text { Reducción de } \\
\text { costos } \\
\text { laborales }\end{array}$ & 35.8 & $\begin{array}{l}\text { Recursos } \\
\text { propios }\end{array}$ & 93.8 & $\begin{array}{l}\text { * Mejora de la } \\
\text { calidad }\end{array}$ & 16 \\
\hline Empleados & 4.9 & $\begin{array}{l}\text { Uso eficiente } \\
\text { de insumos }\end{array}$ & 30.9 & Gobierno & 2.5 & $\begin{array}{l}\text { Búsqueda de } \\
\text { patentes y servicios } \\
\text { tecnológicos }\end{array}$ & 12.3 \\
\hline $\begin{array}{l}\text { Instituciones } \\
\text { (C.I. } \\
\text { universidades) }\end{array}$ & 3.7 & $\begin{array}{l}\text { Aumento en la } \\
\text { escala de } \\
\text { producción }\end{array}$ & 18.5 & Bancos & 2.5 & $\begin{array}{l}\text { * Investigación } \\
\text { con universidades, } \\
\text { Centros de } \\
\text { investigación }\end{array}$ & 8.6 \\
\hline Clientes & 3.7 & & & $\begin{array}{l}\text { Otras } \\
\text { empresas }\end{array}$ & 1.2 & $\begin{array}{l}\text { Comercialización y } \\
\text { exportación }\end{array}$ & 6.2 \\
\hline $\begin{array}{l}\text { Colaboración } \\
\text { terceros para I+D }\end{array}$ & $\begin{array}{c}\text { con } \\
\%\end{array}$ & & & & & & \\
\hline $\begin{array}{l}\text { Universidades } \\
\text { publicas }\end{array}$ & 86.4 & & & & & & \\
\hline Empresas & 8.4 & & & & & & \\
\hline $\begin{array}{l}\text { Centros de } \\
\text { investigación }\end{array}$ & 4.9 & & & & & & \\
\hline
\end{tabular}

* Variables con diferencia significativa entre tamaño de empresas. 
La etapa de adquisición y adaptación de tecnología revela que las Pymes dedican personal a resolver sistemáticamente problemas y a la adaptación y mejora de productos y procesos (así definen la I+D este tipo de empresas). Cerca de la mitad, recluta personal para esta actividad. De la planta actual, poco menos de la mitad tiene estudios superiores (una cuarta parte licenciatura y una quinta posgrado), y en consonancia se tiene la expectativa de emplear preferentemente licenciados a fin de desarrollar primordialmente labores técnicas (menos del cinco por ciento visualizan una dedicación exclusiva a investigación). La mitad de las Pymes cuenta con alguna infraestructura de apoyo, principalmente laboratorios (p. ej. de calidad, análisis o pruebas) y talleres de mantenimiento. Los recursos para investigación provienen de la propia empresa. Cuando se contratan servicios externos se privilegia a la universidad. Así la estrategia alternativa para desarrollar proveedores tecnológicos es a través de sus propios recursos en colaboración con la universidad.

En lo que se refiere al tema de adquisición de tecnología las Pymes operan en su mayoría con maquinaria y equipo moderno, cuatro de cada cinco empresas adquirieron tecnología en los dos últimos años y en su mayoría en nuevas condiciones. La gran mayoría la adquiere mediante transacciones de mercado con proveedores especializados. Son diversos los impactos por la adquisición de tecnología, por orden de importancia, productividad, mejora de proceso, costos y eficiencia. Dos de cada diez empresas han recibido algún tipo de apoyo, preferentemente para modernización, capacitación y productividad, así como para calidad.

La única variable donde se encontraron diferencias significativas por tipo tamaño de empresa fue en la importancia de los apoyos. Así los apoyos de crédito y la asistencia técnica para la modernización, el fondo de cooperación para investigación, normalización y mejora de la calidad y los servicios para búsqueda de patentes e información tecnológica tienen más importancia para las medianas en comparación con las pequeñas, mientras que para las microempresas los problemas que se enfrentan para no utilizarlos son las garantías elevadas que piden.

En la función de la asimilación e implementación del patrimonio tecnológico (FIV) puede destacarse el involucramiento de las Pymes con cambios en los productos, procesos de ingeniería y producción y adaptación (al menos ocho de cada diez). El 75\% comparte con su personal estos cambios a través de diversos medios tales como prácticas, asesoría, consultorías, pero más por capacitación, mientras que a una cuarta parte no le interesa darlos a conocer. La principal fuente para orientar el cambio es el mercado (los clientes y el área de marketing) mientras que la segunda son fuentes internas tales como las áreas de ingeniería e investigación y desarrollo y la tercera del entorno específico - proveedores- y genérico -Internet y la familia-. En este punto existen diferencias significativas, siendo los clientes importantes para todas las Pymes, aún lo son más para las pequeñas y microempresas. Y otro elemento diferenciador para las microempresas es la transcendencia que tienen las fuentes como el Internet y la familia. Finalmente, podemos destacar que la profundidad de los cambios tiene que ver con el propio desempeño histórico de la empresa y no con un nivel de competitividad nacional o internacional. Poco más de una décima parte está realizando modificaciones competitivas a través del registro de patentes y marcas, además de la celebración de contratos de confidencialidad.

En cuanto a su patrimonio tecnológico, siete de cada diez Pymes valorizan su tecnología en el promedio mientras que sólo diez de un ciento la consideran por encima. Esta tecnología se califica por un poco más de la mitad de los respondiente como en la fase de madurez y un tercio en desarrollo, mientras que en ascenso algo más del $10 \%$. 
Tabla 6.

Etapa 4. Asimilación e implementación del patrimonio tecnológico.

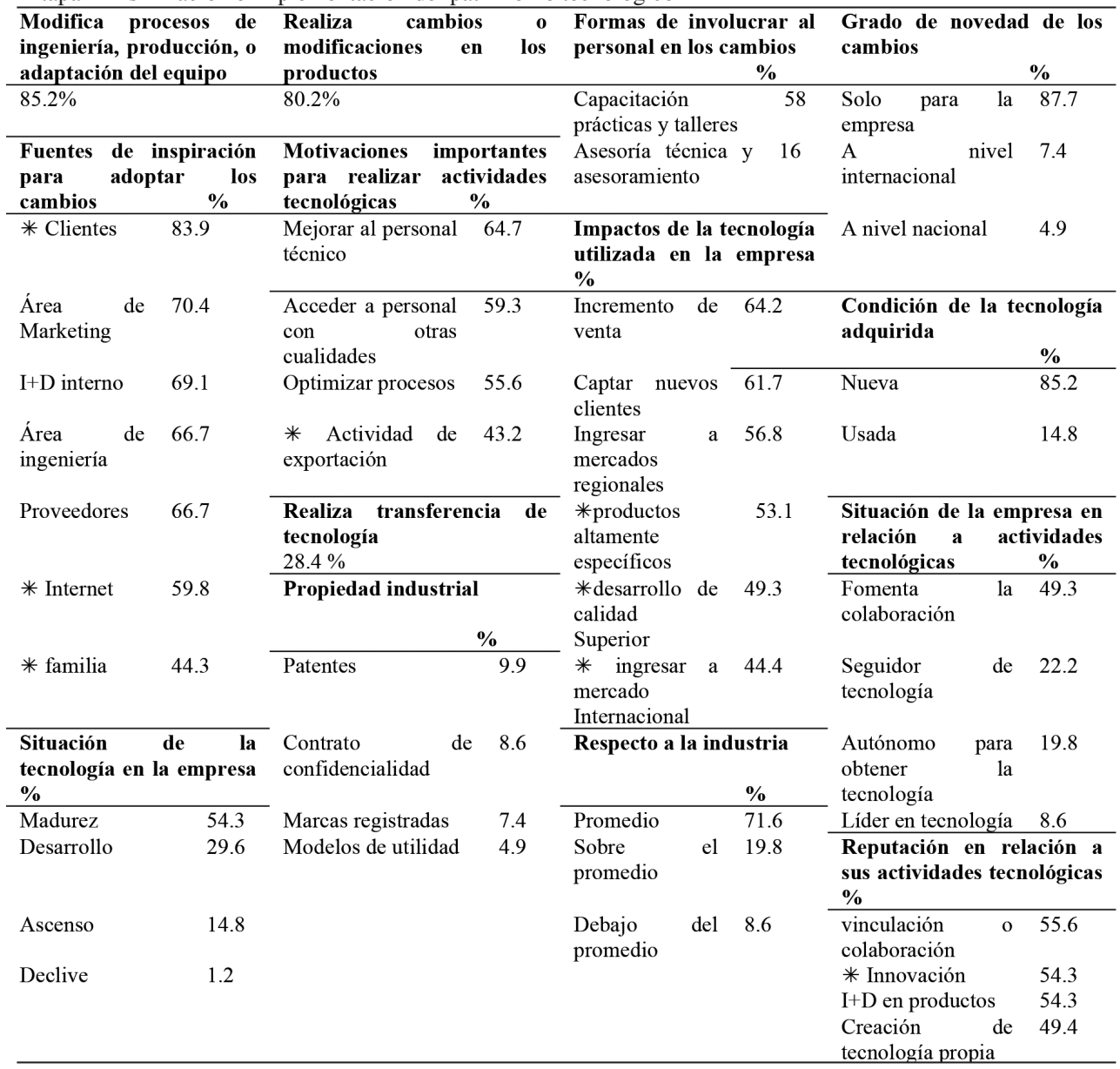

* Variables con diferencia significativa entre tamaño de empresas.

En lo que refiere al impacto que se obtiene por utilizar la tecnología más de la mitad considera que ha sido el incremento de las ventas o captar nuevos clientes o ingresar en mercados regionales, un poco menos orientarse al mercado externo, mientras que un nivel intermedio de Pymes considera que el efecto principal es en los productos, dotarles de especificidad o de calidad. La racionalidad para el desarrollo tecnológico está relacionada con el capital humano, ya sea para mejorarlo o complementarlo o para mejorar los procesos, y un poco menos para fomentar la capacidad exportadora. Este desarrollo tiene un alcance limitado hacia la propia empresa pues sólo un tercio de las empresas declaran que transfieren o comparten los cambios. En cuanto a la estrategia innovadora, menos de un décimo se considera líder, una quinta parte son autónomos y un 50\% son dependientes, pues están subordinados a sus clientes y proveedores para acceder a las innovaciones tecnológicas. Finalmente, lo que las Pymes perciben que sus 
stakeholders valoran más en sus actividades tecnológicas es su capital relacional, la novedad y cambio en los productos, así como sus esfuerzos por mantener su tecnología.

De acuerdo con las pruebas ANOVA realizadas, las Pymes muestran diferencias en cuanto a la racionalidad para el desarrollo tecnológico, los impactos por el uso de tecnología y en su reputación tecnológica. Para la pequeña y mediana la actividad tecnológica puede ser determinante para iniciar o aumentar la actividad exportadora, mientras que sólo para las empresas medianas se puede decir que la tecnología usada ha tenido como impacto entrar a los mercados internacionales. Y un elemento diferenciador es que sólo en las medianas la tecnología ha podido impactar en el desarrollo de productos altamente específicos para nichos especializados y productos de calidad superior en la industria. Lo cual se puede corroborar en la reputación que se hace en el mercado pues la actividad tecnológica genera a las empresas medianas fama de innovadoras.

\section{Patrones por tamaño}

Las microempresas catalogan más importante los canales de abastecimiento y proveedores como fuente de información para analizar el entorno. Para ellas es todavía más importante realizar un estudio técnico antes de adquirir la tecnología, integran mayormente la I+D a las ventas que a cualquier otra área. Se inspiran en los clientes, internet y familiares para realizar cambios. Destinan de sus ventas un porcentaje aún bajo para la compra de nueva tecnología, tratan de proteger los cambios que realizan y aunque pocas solicitan patentes suelen ser reconocidas como empresas innovadoras.

Las pequeñas empresas realizan actividades de análisis del entorno, establecen los criterios para la evaluación y selección de la tecnología. Realizan actividades de investigación y desarrollo, destinan personal a estas actividades, ocupan un porcentaje medio alto en la compra de nuevas tecnologías y han tenido cooperación con otras instituciones para realizar investigación y desarrollo. Realizan cambios y adecuaciones, protegen sus innovaciones. Sin embargo, no son completamente reconocidas como empresas innovadoras.

Las empresas medianas tienen un comportamiento más acelerado, cumplen y realizan todas las fases, actividades y características de una gestión tecnológica eficiente: 1) analizan el entorno, 2) investigan y evalúan la tecnología antes de adquirirla, 3) adquieren la tecnología de acuerdo con las necesidades específicas a satisfacer, realizan cambios y adecuaciones; 4) implementan su tecnología con los cambios realizados además de proteger dichos cambios. Cabe destacar que destinan un porcentaje medio-alto para realizar actividades de investigación y desarrollo y para la compra de nueva tecnología, así como que son percibidas como empresas innovadoras.

\section{Patrones por tamaño, sector y localización}

Los seis clústeres identificados pueden dar origen a patrones específicos por tamaño, sector y localización por lo que se presentan a continuación sus características principales. 
Tabla 7. Principales Diferenciadores en los Patrones Empíricos de Gestión Tecnológica.

\begin{tabular}{|c|c|c|c|c|c|c|}
\hline Cluster & $\mathrm{I} / 10$ casos & II/ 5 casos & $\mathrm{VI} / 40$ casos & V/ 15 casos & III/ 2 casos & IV/ 9 casos \\
\hline Tamaño & Micro & Micro & Micro-pequeña & Micro-pequeña & $\begin{array}{l}\text { Pequeña- } \\
\text { mediana }\end{array}$ & Mediana \\
\hline Sector I & & Construcción & & Manufa & ctura & Agroindustria \\
\hline Sector II & & Manufactura & $\begin{array}{l}\text { Manufactura } \\
+ \text { Agroind }\end{array}$ & & Construcción & Manufactura \\
\hline Localidad & $\begin{array}{l}\text { Irapuato- } \\
\text { Guanajuato }\end{array}$ & $\begin{array}{l}\text { Celaya- } \\
\text { León }\end{array}$ & $\begin{array}{l}\text { Corredor } \\
\text { Industrial }\end{array}$ & $\begin{array}{l}\text { Celaya- } \\
\text { Salamanca }\end{array}$ & $\begin{array}{l}\text { León- } \\
\text { Salamanca }\end{array}$ & $\begin{array}{l}\text { Celaya- } \\
\text { León }\end{array}$ \\
\hline Planeación & $\begin{array}{l}\text { Semestral } \\
\text { Anual }\end{array}$ & Semestral & Anual & $\begin{array}{l}\text { Semestral } \\
\text { Anual }\end{array}$ & Anual & Semestral \\
\hline $\begin{array}{l}\text { Entorno } \\
\text { Genérico } \\
\text { Entorno } \\
\text { relevante }\end{array}$ & $\begin{array}{l}\text { Económico } \\
\text { Tecnológico }\end{array}$ & Económico & Tecnológico & $\begin{array}{l}\text { Ecológico } \\
\text { Económico } \\
\text { Tecnológico }\end{array}$ & $\begin{array}{l}\text { Estudio } \\
\text { técnico** }\end{array}$ & $\begin{array}{l}\text { Económico } \\
\text { Tecnológico } \\
\text { Mercado** } \\
\text { Socios** }\end{array}$ \\
\hline $\begin{array}{l}\text { Criterios de } \\
\text { Adquisición }\end{array}$ & $\begin{array}{l}\text { Estudio } \\
\text { técnico** }\end{array}$ & $\begin{array}{l}\text { Precio } \\
\text { Estudio } \\
\text { técnico** }\end{array}$ & & $\begin{array}{l}\text { Estudio } \\
\text { técnico** }\end{array}$ & & $\begin{array}{l}\text { Estudio } \\
\text { técnico** }\end{array}$ \\
\hline $\begin{array}{l}\text { Condición } \\
\mathrm{I}+\mathrm{D}\end{array}$ & & & & $\mathrm{I}+\mathrm{D}$ formal & & $\mathrm{I}+\mathrm{D}$ formal \\
\hline $\begin{array}{l}\text { Integración } \\
\text { I+D }\end{array}$ & Ventas** & Ventas** & Ventas** & Sin integración & Ventas** & Ventas** \\
\hline $\begin{array}{l}\text { Estudios } \\
\text { personal I+D }\end{array}$ & Graduados & Graduados & Graduados & Graduados & Graduados & Posgrado \\
\hline $\begin{array}{l}\text { Fuente } \\
\text { Adquisición }\end{array}$ & Proveedores & Proveedores & Proveedores & $\begin{array}{l}\text { Empleados } \\
\text { Proveedores }\end{array}$ & Proveedores & Proveedores \\
\hline $\begin{array}{l}\text { Impactos por } \\
\text { Adquisición }\end{array}$ & $\begin{array}{l}\text { Costos } \\
\text { laborales }\end{array}$ & $\begin{array}{l}\text { Cambio en } \\
\text { el proceso }\end{array}$ & $\begin{array}{l}\text { Cambio en el } \\
\text { proceso } \\
\text { Gama de } \\
\text { productos }\end{array}$ & & & $\begin{array}{l}\text { Cambio en } \\
\text { el proceso }\end{array}$ \\
\hline Infraestructura & $\begin{array}{l}\text { Taller mante- } \\
\text { nimiento }\end{array}$ & $\begin{array}{l}\text { Taller mante- } \\
\text { nimiento }\end{array}$ & $\begin{array}{l}\text { Taller } \\
\text { mantenimiento }\end{array}$ & $\begin{array}{l}\text { Taller } \\
\text { mantenimiento } \\
\text { Lab Calidad }\end{array}$ & Lab Calidad & Lab Calidad \\
\hline $\begin{array}{l}\text { Ventas a } \\
\text { compra de }\end{array}$ & $20 \%$ & $10 \%$ & $10 \%$ & $10 \%$ & $20 \%$ & $10-20 \%$ \\
\hline $\begin{array}{l}\text { tecnología } \\
\text { Apoyos }\end{array}$ & Modernización & $\begin{array}{l}\text { Patentes e } \\
\text { información } \\
\text { tecnológica** } \\
\text { Capacitación }\end{array}$ & $\begin{array}{l}\text { Patentes e } \\
\text { información } \\
\text { tecnológica** } \\
\text { prácticas }\end{array}$ & $\begin{array}{l}\text { prácticas } \\
\text { capacitación }\end{array}$ & $\begin{array}{l}\text { Moderni- } \\
\text { zación** } \\
\text { Calidad** } \\
\text { capacitación }\end{array}$ & $\begin{array}{l}\text { Moderniza- } \\
\text { ción** } \\
\text { Coopera- } \\
\text { ción** }\end{array}$ \\
\hline $\begin{array}{l}\text { Comunicación } \\
\text { al personal } \\
\text { Protección } \\
\text { Intelectual }\end{array}$ & Prácticas & $\begin{array}{l}\text { Confidencialidad } \\
\text { Patentes } \\
\text { Marcas }\end{array}$ & $\begin{array}{l}\text { prácticas } \\
\text { capacitación } \\
\text { Modelo de } \\
\text { utilidad }\end{array}$ & $\begin{array}{l}\text { capacitación } \\
\text { Modelo de } \\
\text { utilidad }\end{array}$ & $\begin{array}{l}\text { Confiden- } \\
\text { cialidad }\end{array}$ & $\begin{array}{l}\text { Capacitación } \\
\text { Patentes }\end{array}$ \\
\hline Inspir & t** $*$ & Internet- familia** & & & & \\
\hline $\begin{array}{l}\text { Impacto } \\
\text { Tecnológico }\end{array}$ & $\begin{array}{l}\text { Calidad } \\
\text { superior** }\end{array}$ & $\begin{array}{l}\text { Productos } \\
\text { específicos } \\
\text { Calidad } \\
\text { superior** }\end{array}$ & $\begin{array}{l}\text { Calidad } \\
\text { superior }\end{array}$ & $\begin{array}{l}\text { Calidad } \\
\text { superior** } \\
\text { Productos } \\
\text { específicos }\end{array}$ & $\begin{array}{l}\text { Calidad } \\
\text { superior** }\end{array}$ & $\begin{array}{l}\text { Calidad } \\
\text { superior** } \\
\text { Productos } \\
\text { específicos** } \\
\text { Mercados } \\
\text { internaciona- } \\
\text { les** }\end{array}$ \\
\hline $\begin{array}{l}\text { Situación } \\
\text { tecnológica } \\
\text { respecto } \\
\text { a la industria }\end{array}$ & $\begin{array}{l}\text { Madurez- } \\
\text { promedio }\end{array}$ & $\begin{array}{l}\text { En desarrollo- } \\
\text { promedio }\end{array}$ & $\begin{array}{l}\text { En desarrollo- } \\
\text { promedio }\end{array}$ & $\begin{array}{l}\text { Madurez- } \\
\text { promedio }\end{array}$ & $\begin{array}{l}\text { Madurez- } \\
\text { debajo del } \\
\text { promedio }\end{array}$ & $\begin{array}{l}\text { Madurez- } \\
\text { sobre el } \\
\text { promedio }\end{array}$ \\
\hline $\begin{array}{l}\text { Reputación } \\
\text { tecnológica }\end{array}$ & $\begin{array}{l}\text { Seguidor- } \\
\text { innovador }\end{array}$ & $\begin{array}{l}\text { Seguidor- } \\
\text { innovador }\end{array}$ & Seguidor & $\begin{array}{l}\text { Autónoma- } \\
\text { innovador }\end{array}$ & $\begin{array}{l}\text { Mediana- } \\
\text { mente** }\end{array}$ & $\begin{array}{l}\text { Autónoma- } \\
\text { innovador }\end{array}$ \\
\hline
\end{tabular}

Donde $* *=$ variable estadísticamente significativa en la Prueba de Tukey. 
Las microempresas dan lugar a dos patrones característicos. Las que están ubicadas en Irapuato-Guanajuato (clúster 1, 12.5\%), exclusivamente de la construcción, que se consideran en madurez tecnológica actuando en el promedio de la industria que realizan una planeación tecnológica más frecuente y que pone mayor alcance en el entorno al establecer en su atención al económico y tecnológico. Consideran el principal impacto de la adquisición el disminuir los costos laborales y comunican al personal los cambios mediante prácticas. Dedican hasta el $20 \%$ a la compra de tecnología y su principal apoyo es para la modernización tecnológica. El impacto que destacan por la implementación de la gestión tecnológica es la calidad superior de su producción. Mientras que las que se encuentran en Celaya- León (clúster 2, 6.3\%), de construcción y manufactura, califican la tecnología en uso en desarrollo y que actúan también en el promedio de su industria. Además de considerar el estudio técnico, jerarquizan el precio como principal criterio para la selección de tecnología. Identifican como el principal impacto de la adquisición el cambio en el proceso productivo. Sólo dedican hasta el $10 \%$ a la compra de tecnología y el principal apoyo que solicitan es para la búsqueda de patentes e información tecnológica. El mecanismo para proteger su patrimonio tecnológico va desde patentes y contratos de confidencialidad hasta marcas. Se destaca que entre sus principales fuentes de información además del Internet estén los familiares. Su gestión tecnológica va encaminada a desarrollar productos altamente específicos para sus clientes, amén de su calidad superior.

Las empresas pequeñas presentan tres patrones empíricos de gestión (clúster 6, 5 y 3). Los dos primeros incluyen a empresas micro, que se consideran en una situación tecnológica en promedio en su industria. Ambos comunican los cambios a sus empleados mediante prácticas y capacitación. Aseguran su patrimonio tecnológico mediante los registros de modelos de utilidad. El primero con empresas micro (clúster 6, 49.4\%), está presente en todos los municipios estudiados del corredor industrial y abarca los tres sectores analizados. En su planeación consideran al entorno tecnológico. Su I+D está altamente integrada con la función de ventas. Sus impactos de adquisición se distinguen por incluir el cambio en el proceso y el incremento en la gama de productos. El apoyo requerido es para la búsqueda de patentes e información tecnológica. Se califican como seguidores tecnológicos. Mientras que el segundo patrón con empresas micro (clúster 5, 18.5\%), ubicado en Celaya y Salamanca en el sector de la manufactura, es uno de los más completos al formalizar su I+D. Muestran una planeación frecuente que incluye diversos entornos como el ecológico. Además de los proveedores tecnológicos, consideran a sus empleados como una fuente de adquisición importante. En su infraestructura de adaptación tecnológica incluyen talleres de mantenimiento y laboratorios de calidad. El principal impacto de la adquisición es la productividad y la mejora de los productos. El apoyo más valorado es para la modernización tecnológica. Procuran que su gestión se traduzca en una calidad superior y en productos orientados a los clientes. Y se consideran seguidores tecnológicos con autonomía y perciben una reputación de innovadores. Finalmente, el tercer patrón (clúster 3, 2.5\%) representa a empresas pequeñas y medianas que se califican debajo del promedio industrial y que se ubican en León y Salamanca, tanto en manufactura como en construcción. Pone más énfasis en el entorno relevante en su planeación anual. Evalúa el mercado preponderantemente con estudios técnicos a través de sus graduados que se desempeña en el área de ventas. Dependen de sus proveedores, capacitación y laboratorios de calidad para su adquisición y adaptación tecnológica, a las que aplican hasta un $20 \%$ de sus ventas. Apuestan a resguardar sus activos tecnológicos mediante confidencialidad y los califican de maduros, además de sentirse percibidos como medianamente innovadores por sus stakeholders. 
En cuanto al análisis de clúster, la talla mediana de empresa constituye un patrón empírico (clúster 4, 11.1\%). Se ubica en la agroindustria y la manufactura en los municipios más desarrollados del Estado, Celaya y León. Su planeación es semestral e incluye los entornos económico y tecnológico. Su I+D está formalizada y ocupa personal con posgrado. No sólo requiere apoyos para modernización tecnológica sino también para investigación en cooperación. Se protegen con patentes y declaran que su gestión, además de estar orientada a la calidad superior y a los clientes, procura sustentar el ingreso a mercados internacionales. La situación tecnológica en sus sectores e industria las consideran maduras y se referencian en el promedio. Declaran autonomía tecnológica y estar reputadas como innovadoras.

\section{Discusiones y conclusiones}

Las actividades base para la función del Análisis del Entorno de las Mipymes en nuestro estudio son la realización de análisis situacional interno y externo, con base anual, considerando a los empleados, clientes y proveedores, así como a la maquinaria y equipo privilegiando la información de las noticias de empresas y los canales de abastecimiento.

Esta función puede alinearse con los modelos teóricos en la medida que da cuenta de actividades sistemáticas para captar información -tanto del interior como del exterior de la empresa-, revela la consideración de diversos stakeholders, atención al entorno para buscar oportunidades y amenazas para modernización e innovación tecnológicas.

En cuanto a la investigación de mercado y evaluación de tecnología, nuestra evidencia reconoce una trayectoria de desarrollo propio, donde se valorizan diversas tecnologías de acuerdo con su grado de especialización y sofisticación con criterios de estudio técnico, precio y estudio financiero. La investigación y desarrollo es una competencia distribuida con otras áreas y, en particular, está mayormente integrada con ventas y mantenimiento, aun cuando se considera más importante los departamentos de producción y calidad para el desarrollo de esta actividad.

Esta función revela cierta capacidad para identificar, evaluar y seleccionar tecnología con criterios técnico-económicos donde participan diferentes especialistas de la empresa, pero no necesariamente para diseñar estrategias de aprovechamiento óptimo de la tecnología a adquirir dada la limitada actividad de la I+D como lo establecen los modelos teóricos de gestión.

En la función de adquisición y adaptación tecnológica, la principal fuente de adquisición son los proveedores. A dicha actividad se destina entre el 10 y el $20 \%$ de las ventas y esta inversión busca aumentar la productividad, mejorar la calidad de los productos y realizar cambios en los procesos. La adaptación requiere cambios en los procesos de ingeniería, producción o equipos o modificaciones en los productos, los cuales se llevan a cabo principalmente por graduados, a través de proyectos autofinanciados menores al $1 \%$ de ventas, mismos que aprovechan la infraestructura de los talleres de mantenimiento y laboratorios de calidad. En estas actividades también han recurrido al apoyo de universidades públicas. Y lo que más se demanda son apoyos para la modernización, capacitación y calidad.

La función empírica establece un manejo de recursos internos de forma organizada, con objetivos claros que permiten transitar desde la compra hasta la operación con una financiación propia que faculta la puesta en práctica por el propio personal y, en el caso, auxiliándose de apoyos en el entorno facilitar la asimilación, pero se revela una alta dependencia de los proveedores y muy pocas vinculaciones. 
En las actividades asociadas a la asimilación e implementación de tecnología, el alcance de la novedad de los cambios realizados está circunscrito, básicamente, a la propia empresa y se dan a conocer al personal operativo a través de capacitación y prácticas. Dichos cambios están inspirados por los clientes y el área de mercadotecnia, por la producción y la I+D, los proveedores, así como el Internet y la familia. Coadyuvan a mejorar y acceder al capital humano, optimizar los procesos e incluso en las empresas más grandes a detonar la exportación. Los impactos se reflejan en diversos indicadores de competitividad tales como costos, ventas, penetración, expansión, desarrollo de productos y su calidad, desarrollo de mercados internacionales, entre otros. Pese a que se considera que la industria en promedio utiliza tecnología madura se puede llegar a generar patrimonio tecnológico propio.

En lo que concierne a sus referentes teóricos, nuestros casos muestran la capacidad de generar innovaciones menores a través de la adaptación a sus necesidades específicas. Se perciben diversos impactos por estas actividades en el ámbito tecnológico, económico y emocional. Se establece un patrimonio tecnológico limitado con pocas salvaguardas, pero diversas, desde patentes, modelos de utilidad y marcas hasta contratos de confidencialidad. Pocas empresas participan en la transferencia de sus modificaciones e incluso algunas no les interesa darlas a conocer. El éxito está asociado con mantener los proveedores tecnológicos, modificar productos y conservarse en el promedio tecnológico de la industria.

A partir de los clústeres, se puede inferir la mejor práctica de la gestión tecnológica de las Pymes del sector industrial del estado de Guanajuato. Cabe mencionar que estas son y lo que se pretende al exponerlas es que puedan tener un sentido estratégico donde los empresarios se identifiquen dentro de un clúster, se comparen con los demás, y se propongan una mejora continua de esta actividad para generar y desarrollar las capacidades tecnológicas que sostengan sus resultados en el largo plazo.

Para la fase I de Análisis del Entorno, el instrumento muestra que los seis clústeres son muy parecidos. Los clústeres más limitados son los de microempresas y de construcción (C1, C-2 y C-3) presentado algunas fallas en la vigilancia tecnológica, análisis del entorno y la identificación de fuentes de información. El clúster 4 comprendido por empresas medianas (Celaya-León) y el clúster 5 compuesto por una mezcla entre micro y pequeñas empresas manufactureras (química y textil en Celaya-Salamanca), son los que revelan un mejor puntaje en esta fase.

Dentro de la fase II relativa a la Investigación y Evaluación de la tecnología, el clúster 1 de microempresas constructoras en Irapuato-Guanajuato es el que presenta la mayor deficiencia, en particular con respecto a los proveedores y al área de I+D. Los clústeres de mayor talla, el 3 (de pequeñas y medianas en León-Salamanca) y el 4 (de medianas en Celaya-León), encuadrados en los tres sectores analizados muestran la mejor marca.

En cuanto a la fase III de Adquisición y Adaptación de la Tecnología, el clúster 6 (micropequeñas deslocalizadas) y el 1 (micro constructoras) prestan menor atención a la contratación de personal e infraestructura para la I+D, lo cual los lleva a un deficiente desempeño en la modificación de procesos, pero principalmente de productos, con lo cual son los más limitados en esta fase. El clúster 4 (empresas medianas en Celaya-León) y 3 (pequeñas y medianas en León-Salamanca) presentan el mejor cumplimiento de la fase destacando una mayor inversión a la adquisición tecnológica combinada con programas de asistencia técnica y modernización tecnológica. 
Finalmente, en lo que se refiere a la fase IV de Asimilación e Implementación de la tecnología, el clúster con menor eficiencia relativa fue el de micro y pequeñas empresas deslocalizadas (clúster 6). Se consideran a sí mismas seguidoras tecnológicas y se distinguen por usar modelos de utilidad como mecanismo de salvaguarda y enfocar los impactos de introducción de tecnología en aumentar la gama de productos en una situación donde considera su tecnología en proceso de desarrollo. Pero si consideramos al resto de los clústeres, que utilizan tecnología madura, el del desempeño más limitado es el 1 de microempresas constructoras, las cuales se consideran seguidoras tecnológicas y cuya racionalidad para introducir tecnología es el aumento de escala y la reducción de costos labores. Por otra parte, el patrón que se reveló más sofisticada fue el de las empresas medianas (clúster 4). El alcance de la novedad de sus modificaciones llegó al nivel internacional. El mecanismo para salvaguardar las innovaciones resultaron ser las patentes. Pese a utilizar tecnología madura declararon estar por encima de su industria. La introducción de tecnología buscaba múltiples propósitos desde modificar los procesos y mejorar la calidad hasta aumentar la productividad. Se consideran empresas autónomas y con prestigio de innovadoras, lo que les permite internacionalizar su producción.

Estas prácticas sugieren que hay un mejoramiento tecnológico con el aumento de escala, diferencias en complejidad entre sectores (siendo mayor la derivada de la manufactura frente a la construcción) y entre ubicaciones (mayor en ciudades de mayor desarrollo relativo como León y Celaya), pero también que hay diferencias de complejidad entre los mismos segmentos de talla presentándose dos patrones en cada uno de los tamaños considerados.

Si la innovación sigue una dinámica compleja a partir de procesos graduales y acumulativos entonces podemos presumir un crecimiento basado en una evolución de la sofisticación en la gestión tecnológica. De acuerdo con Nooteboom (1994) las Pymes pueden cambiar de talla para abatir debilidades innovativas tales como una capacidad de absorción limitada, deficiente disponibilidad de información, alta rotación de cuadros calificados, falta de inteligencia tecnológica y mala condición para asumir riesgos.

En cuanto a las diferencias por tamaño, sector y localidad podemos hacer algunas interpretaciones en cuanto a regularidades empíricas con las cuales distinguir ciertos patrones y avanzar a conformar posibles interrelaciones entre los clústeres sobre el grado de sofisticación y el potencial cambio de talla, esto es, no sólo quedarnos con un patrón estático sobre el tamaño sino con base en la sofisticación en la gestión tecnológica postular un patrón dinámico para transitar de un tamaño a otro.

En cuanto al patrón de "complejización-aumento de talla" podemos dibujar una posible trayectoria desde la empresa micro con el patrón más sencillo de gestión tecnológica hacia la empresa mediana con la gestión más compleja. Atendiendo a la función de Análisis del entorno, el patrón se complejiza y la empresa crece por el incremento en la frecuencia en la planeación y complejización del entorno. En lo que se refiere a la Investigación de mercado y evaluación tecnológica, la práctica común es realizar estudios técnicos e integrar la actividad de $\mathrm{I}+\mathrm{D}$ con el área de ventas. En la siguiente función relativa a la Adquisición y adaptación tecnológica, los impactos que experimentan las empresa van desde costos laborales hacia cambios en los procesos, desde la necesidad de apoyos de modernización hacia los de acceso a información tecnológica y de vuelta hacia los de modernización tecnológica (patrón circular), y de aquí hacia la mejora de la calidad y fuentes de cooperación, mientras que la infraestructura de apoyo se soporta desde un taller de mantenimiento hacia un laboratorio de calidad. Finalmente, en la función de Asimilación e implementación, la propiedad intelectual transita 
desde la confidencialidad y marcas a modelos de utilidad y de vuelta a confidencialidad (patrón circular), desde donde se avanza hacia el patentamiento; y el impacto tecnológico pasa por la calidad hasta llegar a productos específicos y terminar en mercado internacionales.

Así, con esta diversidad de patrones podemos considerar que los objetivos de este trabajo de investigación fueron cumplidos. La situación actual de la gestión tecnológica se describió función por función, lo cual reveló una visión estratégica manifestada en una orientación que permite desarrollar capacidades de aprendizaje -identificar, asimilar y explotación de conocimiento del entorno- y capacidad (interna) de gestión de recursos -de forma racional y apropiada: capital, experiencia y tecnología para los procesos de innovación- si bien se queda muy corta en cuanto a otras capacidades de innovación tales como capacidades de I+D, de producción y de vinculación. Con estas descripciones podemos aceptar la proposición elaborada. Las Pymes del sector industrial del estado de Guanajuato realizan algunas funciones de los modelos de gestión tecnológica con visón estratégica por lo que podemos comprobar, con los resultados de la encuesta, las distintas pruebas y técnicas estadísticas que conforman diversos patrones empíricos de la gestión de tecnología de acuerdo con su tamaño, sector y localización, la cual se probó mediante la descripción de los grupos obtenidos por el análisis de clúster.

$\mathrm{Si}$ bien todos los tamaños de empresas analizados realizan las funciones básicas de gestionar tecnología y su entorno relevante las califica como empresas innovadoras se presentan diferencias significativas entre ellas. Los factores que inducen estas diferencias, como lo muestra la literatura el desarrollo y crecimiento de una empresa y lo ratifica nuestra investigación, están marcados por la capacidad de acceso a recursos y su entorno. Pero nuestro estudio hace patente, de que existen estas diferencias además por la sofisticación tecnológica. Situación que afecta los deseos y visiones de los empresarios al momento de querer extender el patrimonio tecnológico. Así, la gestión tecnológica no depende solamente de la compra de nueva y especializada tecnología como erróneamente se tiene pensado. Si no que la diferencia competitiva radica en qué tan eficientemente se gestionan, administran o dirigen los recursos que actualmente se tiene para generar conocimiento, investigación e innovación y así llegar al desarrollo de las capacidades dinámicas de la empresa.

\section{Referencias}

Adecco México (2016) “Pymes en México: experiencias y soluciones”, Disponible en: http://libropyme.adecco.com.mx Anlló, G., Suárez, D. (2008). 2.2. Innovación: Algo más que I+ D. Evidencias Iberoamericanas a partir de las Encuestas de Innovación: Construyendo las Estrategias Empresarias Competitivas. En RICYT (Ed.), El Estado de la Ciencia 2008. (pp. 73-103). Buenos Aires: Red de Indicadores en Ciencia y Tecnología Iberoamericana e Interamericana (RICYT).

Bayraktar, B.A. (1990). On the concepts of technology and management of technology, Proceedings of the Second International Conference on Management of Technology (IAMOT, 1990), Khalil, T.M. y Bayraktar, B.A, eds.: 1161-1175.

BID (2010) Ciencia, Tecnología e Innovación en América Latina y el Caribe. Un compendio estadístico de indicadores. Washington, D.C.: Banco Interamericano de Desarrollo.

Bolukbas, U. y Guneri, A.F. (2016). Technology competency evaluation of SMEs in the machine sub-sector by multi criteria decision making approaches, Proceedings of the 12th International FLINS Conference Uncertainty Modelling in Knowledge Engineering and Decision Making. (pp. 891-897). DOI: https://doi. org/10.1142/9789813146976_0138. 
Bolukbas, U. y Guneri, A.F. (2017, septiembre). Technology competency assessment of enterprises by using different types of clustering, Proceedings of the 14th International Symposium on Operational Research (SOR 2017), Volume 2017, (pp. 302-307).

Bolukbas, U. y Guneri, A.F. (2018). Knowledge-based decision making for the technology competency analysis of manufacturing enterprises. Applied Soft Computing, 67, 781-799. DOI: https://doi.org/10.1016/j.asoc.2017.11.023.

Brunswicker, S. y Vanhaverbeke, W. (2015). Open innovation in small and medium凹sized enterprises (SMEs): External knowledge sourcing strategies and internal organizational facilitators. Journal of Small Business Management, 53(4), 1241-1263. DOI: https://doi.org/10.1111/jsbm.12120.

Bullinger, H., Bauer, W., Ardilio, A. y Seidenstricker, S. (2015, agosto). Technology strategy as enabler for future growth and entering disruptive innovations: An approach for technology-driven SME. 23rd International Conference on Production Research (ICPR 2015). International Foundation for Production Research (IFPR).

Burggräf, P., Dannapfel, M., Bertling, M. y Xu, T. (2018, agosto). Return on CPS (RoCPS): An Evaluation Model to Assess the Cost Effectiveness of Cyber-Physical Systems for Small and Medium-Sized Enterprises. 2018 Portland International Conference on Management of Engineering and Technology (PICMET 2018) (pp. 1-9). IEEE. DOI: https://doi.org/10.23919/PICMET.2018.8481980.

Cant, M.C., Wiid, J.A. y Kallier, S.M. (2015). Product strategy: Factors that influence product strategy decisions of SMEs in South Africa. Journal of Applied Business Research, 31(2), 621-630. DOI: https://doi.org/10.19030/jabr. v31i2.9158.

Cobbenhagen, J. (1999). Managing innovation at the company level: a study on non-sector-specific success factors. Doctoral thesis, Maastricht: Universiteit Maastricht.

Cotec. (1998). Temaguide: Pautas metodológicas en gestión de la tecnología y de la innovación para las empresas. Tomo 2 [Libro en línea]. Madrid: Fundación Cotec para la Innovación Tecnológica. Disponible en: http:// aprendeenlinea.udea.edu.co/lms/extension/pluginfile.php/28261/mod_resource/content/0/herramientas_MODULOII_COTEC.pdf

Cuevas Pacheco, G., León-Sánchez, M.M. y Ramírez, A. (s.f.). Modelo de Integración de la Gestión Tecnológica y Desarrollo de la Gestión a la Innovación de las Pymes. COMECYT. Innovación y Competitividad. Publicación Trimestral de la Asociación de Mexicana de Directivos de la Investigación Aplicada y el Desarrollo Tecnológico (ADIAT).

Deranek, K., Chopra, S. y Mosher, G. A. (2017). Lean Adoption in a Small and Medium Enterprise: Model Validation. The Journal of Technology, Management, and Applied Engineering, 33(3), 2-13.

Faloh Bejarano, R. (Ed.). (2006). Gestión de la innovación: una visión actualizada para el contexto iberoamericano. La Habana: Academia.

Farid, F. (2017). SME sector backbone of economy. Disponible en: https://pakobserver.net/sme-sector-backbone-of-economy/ Acceso el 09 de octubre del 2017.

Filson, A., Lewis, A. (2000). Cultural issues in implementing changes to new product development process in a small to medium sized enterprise (SME). Journal of Engineering Design, 11(2), 149-157. DOI: https://doi. org/10.1080/09544820050034240.

Freel, M.S. (2000). Barriers to product innovation in small manufacturing firms. International Small Business Journal, 18 (2), 60-79. DOI: https://doi.org/10.1177/0266242600182003.

Garengo, P. (2018). How bridging organisations manage technology transfer in SMEs: an empirical investigation. Technology Analysis \& Strategic Management, 1-15. DOI: https://doi.org/10.1080/09537325.2018.1520976.

Hidalgo Nuchera, A. (1999). La gestión de la tecnología como factor estratégico de la competitividad industrial. Economía industrial, (330), 43-54.

Hidalgo, A.; León, G., Pavón, A. (2002). La gestión de la innovación y la tecnología en las organizaciones. Madrid: Pirámide.

Hills, M. y Atkinson, L. (2016). Towards cyber-resilient \& sustainable SMES: the case study of added value from a large IT reseller. En M. Hills, (Ed.), Why Cyber Security is a Socio-Technical Challenge: New Concepts and Practical Measures to Enhance Detection. (Pp. 71-80). Hauppauge, NY: Nova Science Publishers, Inc. 
Lohmüller, B. y Petrikhin, A. (2018, junio). The Growing Importance of Technology Executives/Hidden Chief Technology Officers and Their Organizational Roles. 2018 IEEE International Conference on Engineering, Technology and Innovation (ICE/ITMC) (pp. 1-8). IEEE. DOI: https://doi.org/10.1109/ICE.2018.8436317.

Loon, M. y Chik, R. (2018). Efficiency-centered, innovation-enabling business models of high tech SMEs: Evidence from Hong Kong. Asia Pacific Journal of Management, 1-25. DOI: https://doi.org/10.1007/s10490-017-9558-4.

Malaver, F., Vargas, M. (2004). Los procesos de innovación en América Latina: aportes para su caracterización. Academia Revista Latinoamericana de Administración, (33), 5-33.

Marin, G., Marzucchi, A. y Zoboli, R. (2015). SMEs and barriers to Eco-innovation in the EU: exploring different firm profiles. Journal of Evolutionary Economics, 25(3), 671-705. DOI: https://doi.org/10.1007/s00191-015-0407-7.

Marins, L., Anlló, G. y Schaaper, M. (2012), 2.2. Estadísticas de Innovación: El Desafío de la Comparabilidad. En RICYT (Ed.), El Estado de la Ciencia 2012. (pp. 65-79). Buenos Aires: Red de Indicadores en Ciencia y Tecnología Iberoamericana e Interamericana (RICYT).

Medellín, E.A. (2010). Gestión tecnológica en empresas innovadoras mexicanas. Innovation \& Management Review, 7 (3), 58-78. DOI: http://dx.doi.org/10.5585/rai.2010684.

Mendoza León, J. G., Valenzuela Valenzuela, A. (2014). Aprendizaje, innovación y gestión tecnológica en la pequeña empresa: Un estudio de las industrias metalmecánica y de tecnologías de información en Sonora. Contaduría y administración, 59(4), 253-284. DOI: http://dx.doi.org/10.22201/fca.24488410e.2014.93.

Niaki, M.K. y Nonino, F. (2017). Impact of additive manufacturing on business competitiveness: A multiple case study. Journal of Manufacturing Technology Management, 28(1), 56-74. DOI: https://doi.org/10.1108/JMTM01-2016-0001.

Noh, H. y Lee, S. (2015). Perceptual factors affecting the tendency to collaboration in SMEs: Perceived importance of collaboration modes and partners. Journal of Technology Management \& Innovation, 10(3), 18-31. DOI: http:// dx.doi.org/10.4067/S0718-27242015000300003.

Nooteboom, B. (1994). Innovation and Diffusion in Small Firms: Theory and Evidence. Small Business Economics, 6 , 327-347. DOI: https://doi.org/10.1007/BF01065137.

Nordin, N. y Adegoke, O. (2015). Learning from ERP implementation: A case study of issues and challenges in technology management. Jurnal Teknologi, 74 (1), 57-62. DOI: https://doi.org/10.11113/jt.v74.3369.

Othman Idrissia, M., Amaraa, N. y Landrya, R. (2012). SMEs' degree of openness: the case of manufacturing industries. Journal of Technology Management \& Innovation, 7(1), 186-210. DOI: http://dx.doi.org/10.4067/S071827242012000100013.

Peirano, F. (2007), Technological Change in the Manufacturing Sectors of Argentina and Brazil: An Analysis based on the Innovation Surveys. En J.A. de Negri y L.M. Turchi (Ed.), Technological Innovation in Brazilian and Argentine Firms. (pp. 93-129). Brasilia: IPEA.

Premio Nacional de Tecnología e Innovación. Modelo de Gestión de la Tecnología y la Innovación. 2012. Disponible en http://www.pnt.org.mx/index.php?option=com_content\&view=article\&id=42\&Itemid=18 / Acceso el 18 de abril de 2013.

Scozzi B., Garavelli, C., Crowston, K. (2005). Methods for modeling and supporting innovation processes in SMEs. European Journal of Innovation Management, 8 (1), 120-137. DOI: https://doi.org/10.1108/14601060510578619.

Sila, I. y Dobni, D. (2012). Patterns of B2B e-commerce usage in SMEs. Industrial Management \& Data Systems, 112(8), 1255-1271. DOI: https://doi.org/10.1108/02635571211264654.

Sumanth, D.J., Sumanth, J.J. (1996). The `technology cycle’ approach to technology management. En G.H. Gaynor (Ed.), Handbook of Technology Management. (pp. 3.1-3.17). New York, NY: McGraw-Hill.

Van Der Veen E.J., Perez G. y Sabbatinelli B. (2016, septiembre). Innovation and R\&D at OHB system: Innovation from an upcoming Lsi's point of view. Proceedings of the 67th International Astronautical Congress, IAC 2016.

Vossen, R. (1998). Relative strengths and weaknesses of small firms in innovation. International Small Business Journal, 16(3), 88-94. DOI: https://doi.org/10.1177/0266242698163005.

Zhu, Q., Zou, F. y Zhang, P. (2018). The role of innovation for performance improvement through corporate social responsibility practices among small and medium\sized suppliers in China. Corporate Social Responsibility and Environmental Management. DOI: https://doi.org/10.1002/csr.1686. 